\title{
Analysing the Metro Cityring in Copenhagen
}

\author{
A. H. Kaas \& E. M. Jacobsen \\ Atkins, Denmark
}

\section{Abstract}

When planning new infrastructure it is important that the infrastructure is well suited for the planned operation. This is especially important in case of high frequent operation underground (e.g. metro systems) since it is too expensive to modify the infrastructure later.

This paper describes the importance of adapting new infrastructure to the future operation. This will be done based on analyses carried out for the planned ring metro in Copenhagen. The analyses include:

- Different operation alternatives depending on the running time and the number of trains in operation

- The necessity of withdrawing rolling stock in case of problems

- Changes in the frequency during the day

- The stability of the system

The analyses have shown that the possibility to withdraw rolling stock in case of problems and the possibility of changing the frequency is sensitive to the scheduled plan of operation. This is because the possibilities of withdrawing rolling stock and changing the frequency influence the stability of the system.

Altogether, this paper demonstrates that the use of micro simulation in the planning phase of new infrastructure can improve the later operation. This is because it is possible to evaluate the future operation and the need for improved infrastructure.

Keywords: railway capacity, simulation, vehicle allocation, railway operation, metro, Copenhagen, Denmark, railway, plan of operation, service frequency.

\section{Introduction}

The first Metro line in Copenhagen was opened in 2002. Now, in 2008, the system consists of the two lines M1 and M2 (see figure 1 below) and the politicians have decided to extend the Metro system, with a new City Circle Line 
(Cityringen). This extension is planned to be $15 \mathrm{~km}$ long and have 17 underground stations. A round trip on the City Circle Line is expected to take approximately 24 minutes, but the longest travel time between two stations will be no more than 14 minutes.

The City Circle Line will be serviced by two lines: Line M3 that stops at all stations on the line and line M4 that is a shuttle line between Copenhagen Central Station (in figure 1 same as København $\mathrm{H}$ ) and Nørrebro Station via Østerport Station. The tunnel will be prepared for further extensions of the shuttle line towards Sydhavn and Brønshøj.

In the peak hours, the stations between Copenhagen Central Station and Nørrebro Station (via Østerport) will be served by a train every 100 seconds in each direction. Stations on the outer part of circle will be serviced by a train every 200 seconds.

The entire infrastructure is underground, why later changes are extremely expensive. Therefore, it is very important that the new infrastructure will match the planed operation. This match is ensured by thorough analyses using micro simulation tools.

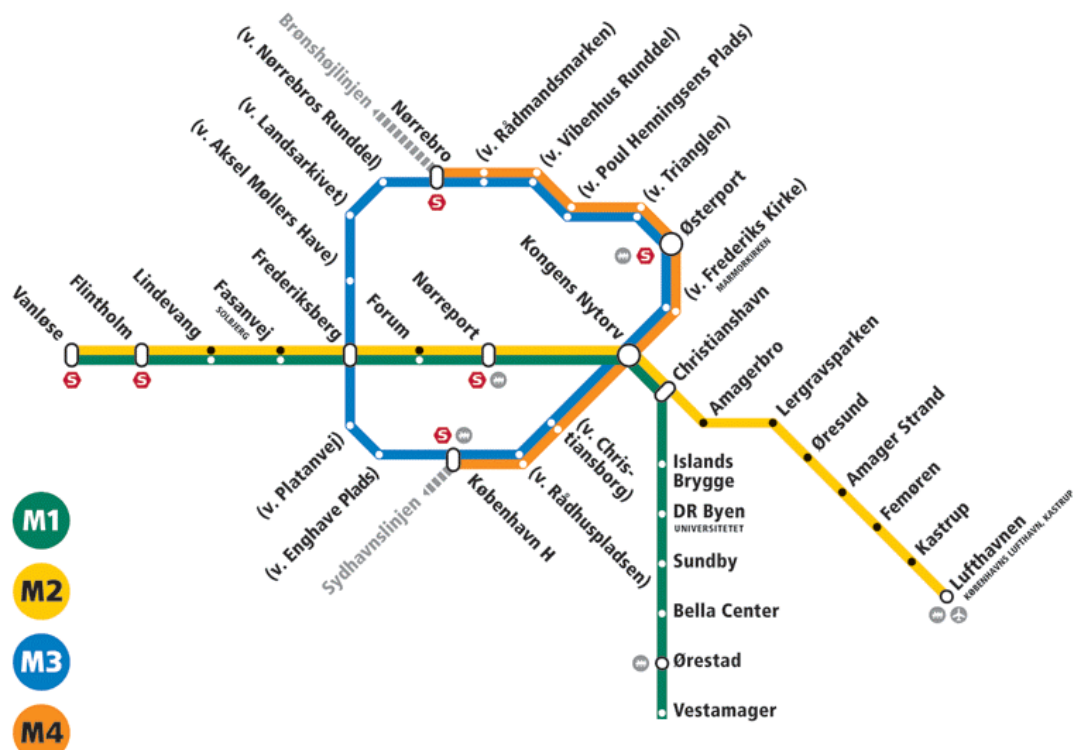

Figure 1: $\quad$ The Copenhagen Cityring.

Analyses of the City Circle Line have been worked out in RailSys and the rostering planning system DISPO. These analyses verify that the planned infrastructure match to the future operation. This approach leads to the following analyses:

- Runtime in the system

- Number of trains in operation 
- Different alternatives of operation

- Train capacity, service frequency and stability

The analyses of the City Circle Line diverge from traditional analyses of railway projects by not taking the amount of delays into account. Instead, the service frequency and the variation of runtime are examined.

The purpose of the analyses is, in the early project phases, to ensure and document that the planned traffic can be operated when the Metro has been constructed.

\section{Analyses}

\subsection{Running time}

The running time on the City Circle Line is a central parameter for the economic and socio-economic analyses. This is because the running time affects the number of trains for the operation alternatives and that lower running time (and thereby more passengers and fewer trains in operation) may justify a higher construction cost.

The running time is calculated based on the planned track layout, distances between stations, gradients, and the expected speed profile.

The characteristic of the rolling stock must take into account that many passengers are standing inside the train since the maximum acceleration and breaking rate then is reduced. Figure 2 shows one of the train types used for the analyses. By implementing these characteristic of the rolling stock and the track layout in RailSys, it possible to calculate the runtime very precise.

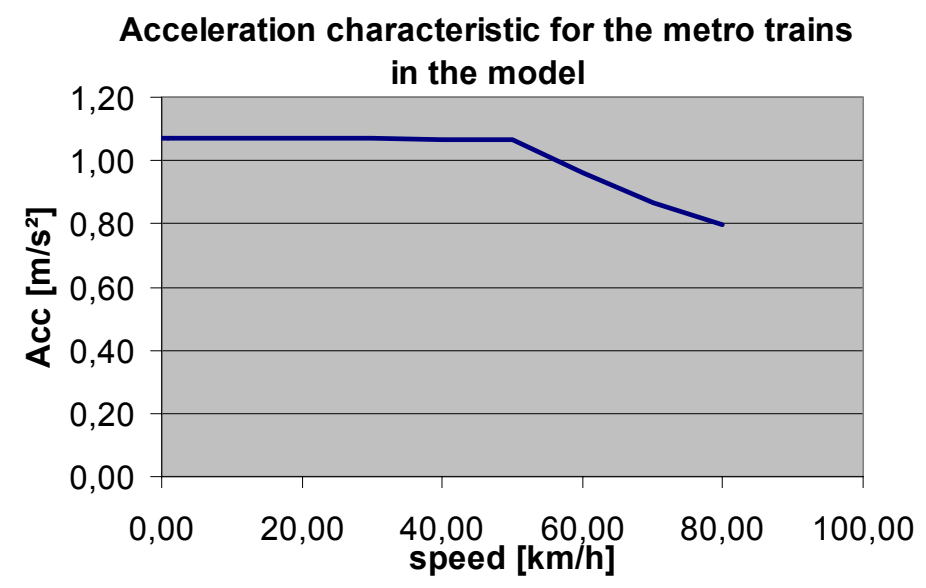

Figure 2: Acceleration characteristic for the metro trains in the model.

In the analyses a number of different speed profiles have been tested to ensure that the running time in the metro is consistent with to the expected running time. Figure 3 shows some of the characteristic of the alignment: Speed restrictions 
around the stations and line speed up to $90 \mathrm{~km} / \mathrm{h}$ between the stations. The stations will be constructed closer to the surface (on so-called "hills"), which make a short access for the passengers and help the metro trains accelerating and decelerating.

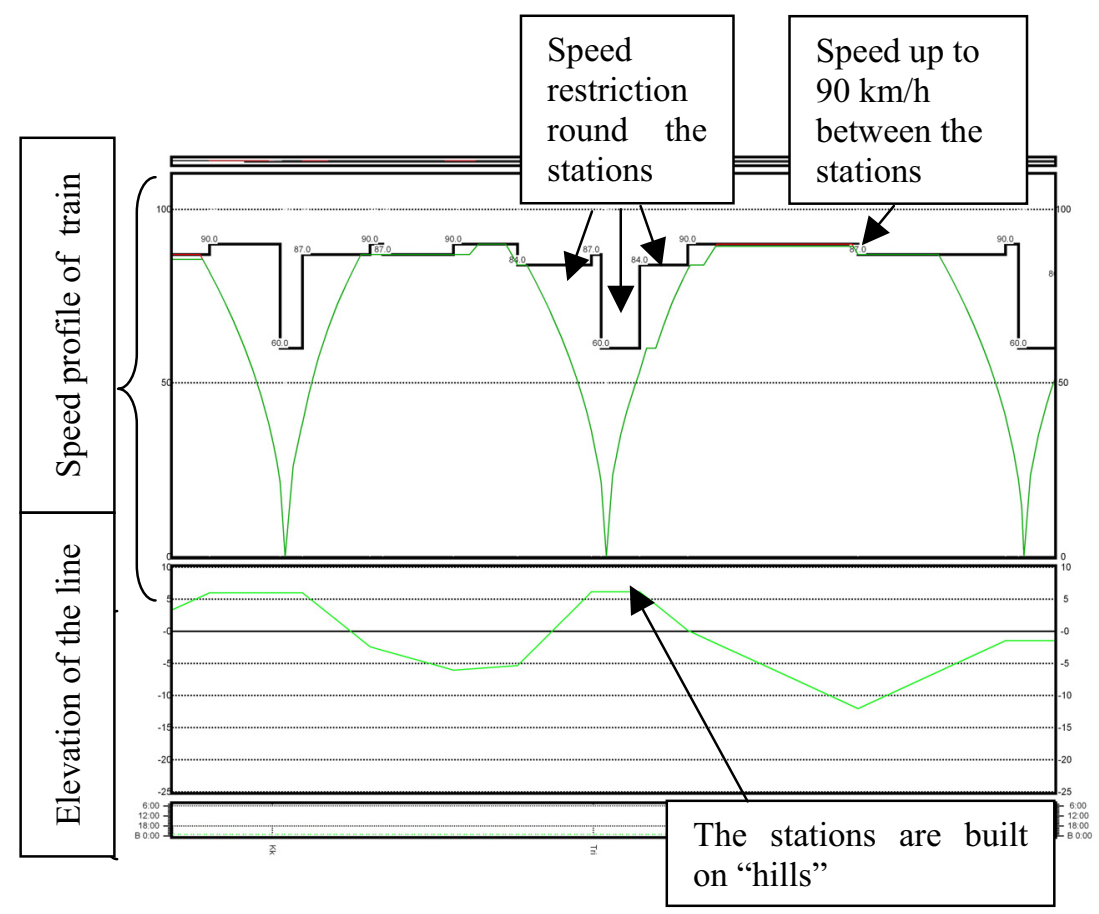

Figure 3: Characteristics of the metro.

The running times, the service frequency of the trains, the dwell times at the stations and in the turn around facilities at the line end stations are core inputs to calculate of the number of trains in operation.

The service frequency is a demand for the system, but it is also a demand to run a fast as possible. Therefore, the service frequency is determined by the number of trains in operation. Many analyses start from a certain number of trains in circulation - e.g. the possible service frequency with 10,15 or 26 trains in service

By using DISPO, it is possible to calculate train kilometres, number of train in deferent scenarios of operation.

\subsection{Operation}

Depending on service frequency and the number of trains in circulation, the metro can be operated by two different principles:

- "Independent". Line M3 is operated as a circular line while line M4 is operated as a shuttle line. Both lines are operated independently in both directions 
- "Butterfly". In the butterfly operation, the lines are mixed. First line M3 is operated as a circular line but when the circle is completed then the line continues as line M4 to its line end station. In this way the train is operated in a $1 \frac{1}{2}$ time circulation before it turns around, cf. figure 4 .

1

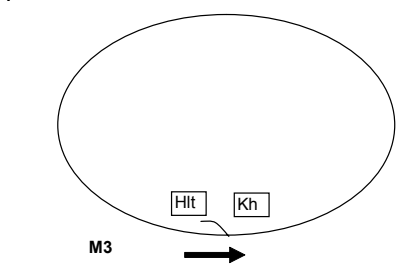

M3 starts from the siding at HIt to Kh and runs round the circle and back to $\mathrm{Kh}$

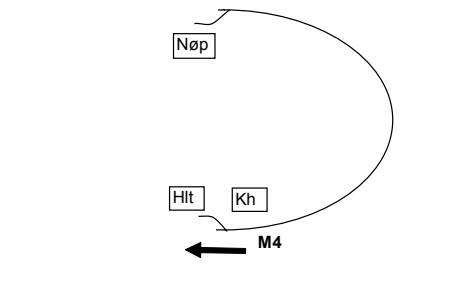

In Nøp the train turn around and return back to Kh as M4
2

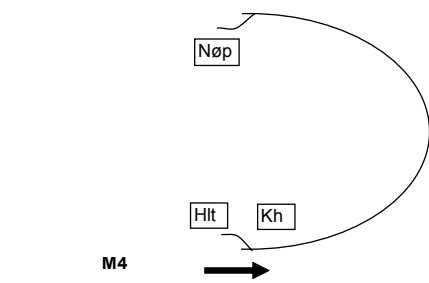

In Kh the train continues as M4 to siding at Nøp

4

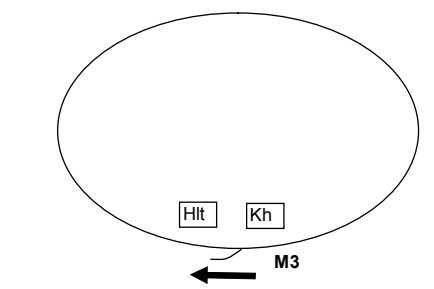

In Kh the train continues as M3 round the circle and back to the beginning at HIt

Figure 4: The "butterfly" principle.

The independent operation will be chosen when the running time of a turnround the circle is (almost) a multiple of the wanted headway. On the other hand, the butterfly operation will be chosen when the running time of a turn-round the circle is (more or less) a multiple of the wanted headway PLUS half the wanted headway. In cases in between the independent and the butterfly operation, the service headway must adapt to one of the operation modes.

\subsection{Service frequency}

RailSys can simulate the theoretical perfect traffic without disturbance. In this it is possible to examine if the planned operation is possible and how the operation will be affected by errors and disturbances. To examine the quality of the operation the following scenarios have been simulated:

- Theoretical perfect traffic (no disturbances).

- Operation where the dwell time have been extended at three stations.

- Operation where trains in one direction run slower than in the other direction.

- Operation where the whole system runs slower.

- Operation where a defect train (only $80 \%$ of normal traction effort) is operated between other trains.

- Operation where a track is blocked between one or more crossovers. 
The quality of the operation is measured by the deviation from the planned service frequency and/or the travel time.

The planned service frequency at Nørrebro station will be 17 to 18 trains per hour (200 - 211 seconds headway), cf. figure 5. This service frequency can be used as a basis when examining disruptions such as dwell time extensions.

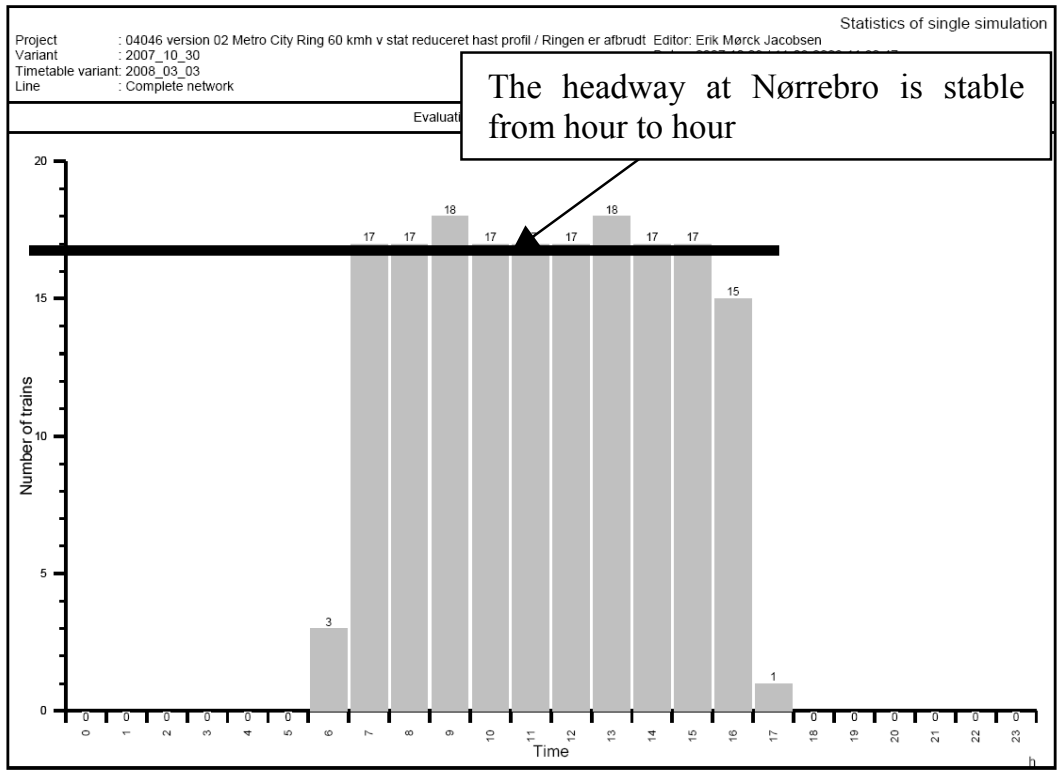

Figure 5: $\quad$ In case of no disturbances, Nørrebro station is serviced by 17 or 18 trains each hour.

Using RailSys, it is possible to examine a dwell time extension in the rush hour. The dwell time is extended from 25 seconds to 45 seconds at three central stations in both directions. The running time round the circle is then 3 times 20 seconds (equal to 1 minute) longer, and the headway will find a new level. At Nørrebro station, the service frequency will stabilize at 16 trains each hour in each direction. However, the first 8 hours, the service frequency will find a new level between 13 trains per hour ( 277 seconds headway) and 16 trains per hour (225 seconds headway).

The example in figure 6 shows that the service frequency will become lower in case of dwell time extensions. However, this is an optimal case as the disturbance affects both directions. In case that the disturbance only affects one direction, the two rings will be out of phase, could cause problems because of the shuttle line (M4), cf. figure 1.

The running time round the circle is 3 times 20 seconds (equal to 1 minute) longer in one direction than the other. By simulating the operation in RailSys, it can be seen that the service frequency is somehow stable at 16 trains each hour, cf. figure 7 . 


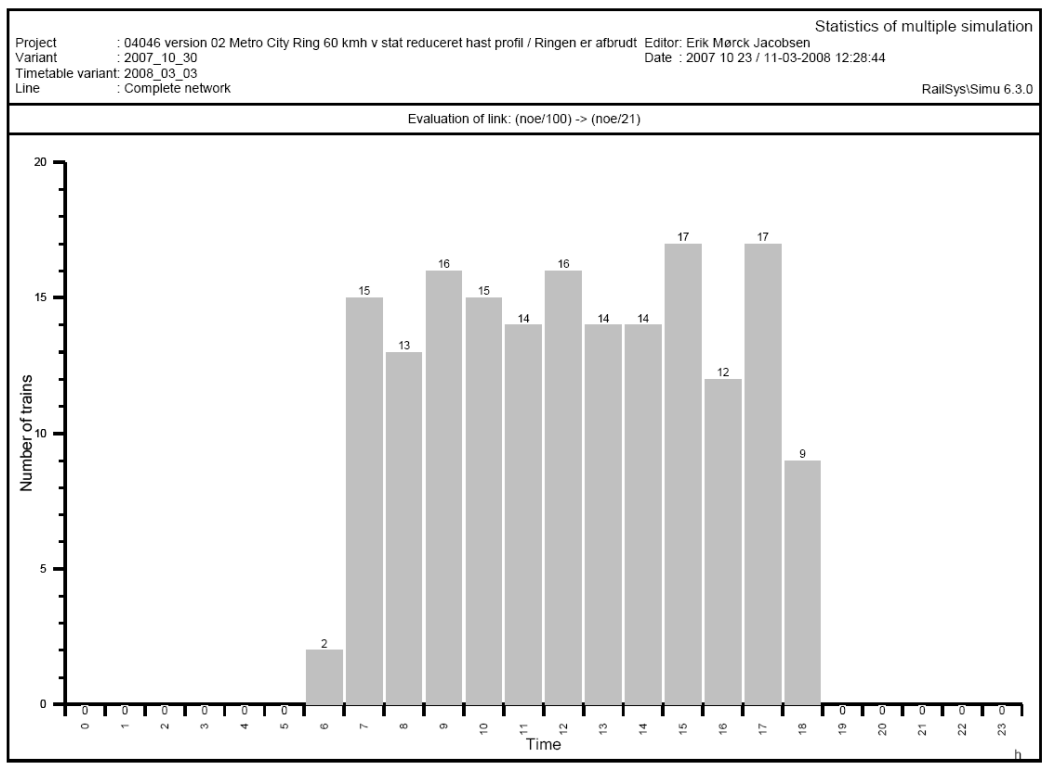

Figure 6: Number of trains departing from Nørrebro in the analyzed case (disturbance in both directions).

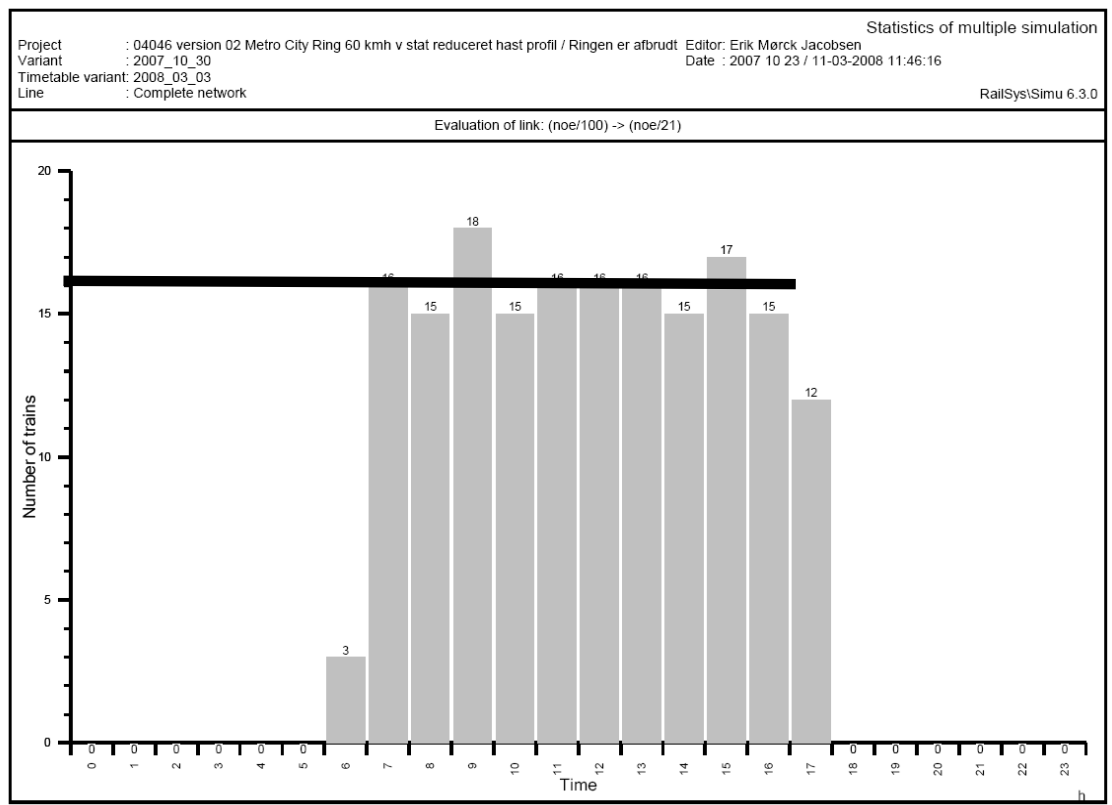

Figure 7: Number of trains departing from Nørrebro in the analyzed case (disturbance in one direction). 
With use of RailSys, it is possible to examine how a train with $20 \%$ reduced traction effort will affect the operation. In this case, the Control Centre must take action and pull the train out. Otherwise, the operation will become unstable with trains queuing up behind the slow train. This unstable situation can be seen from the simulations in figure 8. Here, the service frequency varies at Nørrebro station depending on when the queue of trains passes the station.

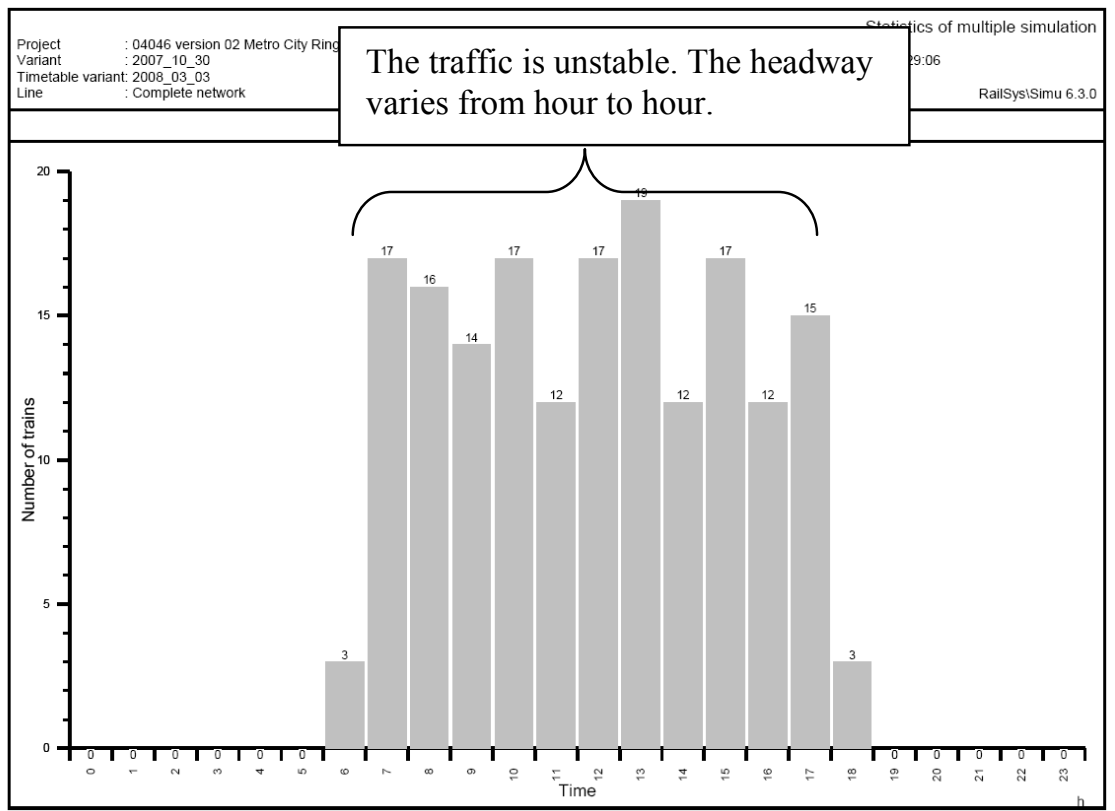

Figure 8: Number of trains departing from Nørrebro in the analyzed case (disturbance of a train with $20 \%$ reduced traction effort).

\section{Conclusion}

Traffic simulation of the City Circle Line gives the possibility to test and document the consequence of different solutions in an early planning phase. In this way it can be ensured that the operation demand matches to the final system.

The analyses show that the service frequency is robust in the cases where all train are affected in the same way: Longer dwell time on some stations, speed restriction etc. In that case the run time will be longer, the headway - and thereby service frequency - finds a new level.

Despite a stable service frequency in case of disturbance, it seems that the operation becomes unstable if the running characteristic of one train in the system differs too much from the others. In that case, the Control Centre must take action and pull the train out as fast as possible. 


\section{References}

[1] Kaas, A.H., Methods to calculate capacity of railways, PhD. thesis at Department of Planning, Technical University of Denmark, 1998 (in Danish).

[2] Kaas, A.H., Punctuality model for railways. Proc. of the 7th International conference on Computers in Railways, eds. J. Allan, R.J. Hill, C.A. Brebbia, G. Sciutto \& S. Sone, pp. 853-860, 2000

[3] Kaas, A.H. \& Goossmann, R., Implementation of the Timetable Planning System STRAX/TPS in Denmark. Proc. of the 9th International conference on Computers in railways, eds. J. Allan, C.A. Brebbia, R.J. Hill, G. Sciutto \& S. Sone, pp. 93-102, 2004

[4] Landex, A. \& Kaas, A.H., Planning the most suitable travel speed for high frequency railway lines. Proc. of the 1st International Seminar on Railway Operations Modelling and Analysis, eds. I.A. Hansen, F.M. Dekking, R.M.P. Goverde, B. Hindergott, L.E. Meester, The Netherlands, 2005 